\title{
Foreword to the Special Focus on Advances in Symbolic and Numeric Computation III
}

\author{
Amélia Loja • Paulo Vasconcelos • Joaquim Infante Barbosa · José Alberto Rodrigues
}

Published online: 13 June 2020

(C) Springer Nature Switzerland AG 2020

The present Special Focus of Mathematics in Computer Science is connected to SYMCOMP2019, the 4th International Conference on Numerical and Symbolic Computation: Developments and Applications, which was held on April 11-12, 2019, in Porto, Portugal. This ECCOMAS Thematic Conference was a joint organization of IDMEC (Mechanical Engineering Institute) and ISEP (Institute of Engineering of Porto), under the auspice of APMTAC (Portuguese Society of Theoretical, Applied and Computational Mechanics).

The conference was also sponsored by Wolfram Research which distinguished two Young Researchers for their relevant works.

The conference proceedings book is published on-line as a 618-page .pdf file at ECCOMAS website, being accessible at https://www.eccomas.org/publications/conference-proceedings/.

Most of the papers in this special focus are extended or new versions of communications presented at the 4th International Conference on Numerical and Symbolic Computation, and reflect its multidisciplinary character. Numerical and symbolic computation methods and techniques are recognised as central tools in numerous fields of science and technology and it is with this perspective that the ten papers included on this Special Focus were selected.

A. Loja $(\bowtie) \cdot$ J. I. Barbosa · J. A. Rodrigues

CIMOSM, Centro de Investigação em Modelação e Optimização de Sistemas Multifuncionais, Instituto Superior de Engenharia de Lisboa, Av. Conselheiro Emídio Navarro, 1, 1959-007 Lisbon, Portugal

e-mail: amelia.loja@isel.pt; amelialoja@tecnico.ulisboa.pt

J. I. Barbosa

e-mail: joaquim.barbosa@isel.pt; joaquim.barbosa@tecnico.ulisboa.pt

A. Rodrigues

e-mail: jose.rodrigues@isel.pt

A. Loja · J. I. Barbosa

IDMEC, Instituto Superior Técnico, Universidade de Lisboa, Avenida Rovisco Pais 1, 1049-001 Lisbon, Portugal

P. Vasconcelos

Centro de Matemática, Universidade do Porto, Rua do Campo Alegre 687, 4169-007 Porto, Portugal

P. Vasconcelos

Faculdade de Economia, Universidade do Porto, Rua Dr. Roberto Frias, s/n, 4200-464 Porto, Portugal

e-mail: pjv@fe.up.pt 
The main topics presented in these papers are summarized next:

\section{Common Points Between Perturbed Chebyshev Polynomials of Second Kind}

\section{Zélia da Rocha}

This paper considers some perturbation of the Chebyshev polynomials of second kind obtained by modifying one of its recurrence coefficients at an arbitrary order. The goal of the work is to point out that perturbed Chebyshev polynomials of fixed degree and different values of parameters of perturbation have some common points, simple or double, that are zeros of two Chebyshev polynomials of second kind of lower degrees. The paper identifies the different cases.

\section{On a 2-Orthogonal Polynomial Sequence via Quadratic Decomposition}

\section{Teresa Augusta Mesquita}

In this work a symbolic approach of the general quadratic decomposition of polynomial sequences is applied to polynomial sequences fulfilling specific orthogonal conditions towards two given functionals $u 0, u l$ belonging to the dual of the vector space of polynomials with coefficients in $\mathrm{C}$. The general quadratic decomposition produces four new sets of polynomials whose properties are investigated with the help of the mentioned symbolic approach together with further commands which inquire relevant features, as for instance, the classical character of a polynomial sequence. The computational results are detailed for a wide range of choices of parameters and co-recursive type polynomial sequences are also explored.

\section{Introducing a New Two-Parameter Invariant Function For Algebras}

\section{J. M. Escobar, J. Núñez-Valdés and P. Pérez-Fernández}

This paper presents the process of construction of a new invariant function of algebras, particularly focusing Lie and Malcev algebras. Currently, the research on invariants of certain algebraic structures is suffering a significant expansion because they allow researchers to take steps forward in the study of those structures, especially those oriented to address the classification problem of algebras. Starting from a paper published by Hrivnák and Novotný in 2007 on invariant functions of algebras the authors present the mentioned construction process and also show main applications

\section{Explicit formulae for integro-differential operational matrices}

\section{José M. A. Matos, Maria João Rodrigues and João Carrilho de Matos}

The article presents the deduction of explicit formulae for the elements of the matrices representing the action of integro-differential operators over the coefficients of generalized Fourier series. The authors' formulae are obtained by performing operations on the bases of orthogonal polynomials and result directly from the three-term recurrence relation satisfied by the polynomials. Moreover they give exact formulae for the coefficients for some families of orthogonal polynomials. The robustness of the formulae presented is illustrated through a set of tests, producing highly accurate results, even when dealing with powers of high dimensional matrices.

\section{Symbolic Computation Applied to the Study of the Kernel of Special Classes of Paired Singular Integral Operators}

\section{Ana C. Conceição}

The main goal of this paper is to show how the symbolic and numeric capabilities of a computer algebra system can be used to study the kernel of special classes of paired singular integral operators with essentially bounded coefficients defined on the unit circle. It is described how some factorization algorithms can be used to compute the dimension of the kernel of special classes of singular integral operators. The analytical algorithms [ADimKerPairedScalar], [AKerPaired-Scalar], and [ADimKerPaired-Matrix] are presented. The design of these new algorithms was focused on the possibility of implementing on a computer, all the extensive symbolic and numeric calculations present in the algorithms. For the essentially bounded Hermitian coefficients case, there exist some relations with Hankel operators. The paper contains also a set of nontrivial examples obtained through the use of a computer algebra system. 


\section{Global Stability of a Caputo Fractional SIRS Model with General Incidence Rate}

\section{Moulay Rchid Sidi Ammi, Mostafa Tahiri and Delfim F. M. Torres}

The present contribution is focused on the use of fractional order derivatives to help reducing errors arising from the neglected parameters in modelling real life phenomena. To this purpose, this paper introduces a fractional order SIRS epidemic model with non-linear incidence rate. The existence of a unique positive solution to the model is proved and the stability analysis of the disease free equilibrium and positive fixed points are investigated. A numerical example is also presented. According to the authors' theoretical analysis, the fractional order parameter $\alpha$ has no effect on the stability of free and endemic equilibria, but it can affect the time for arriving at the steady states.

\section{Synchronization and Self-organization in Complex Networks for a Tuberculosis Model}

\section{Cristiana J. Silvaand Guillaume Cantin}

This paper proposes and analyses the dynamics of a complex network built with non-identical instances of a tuberculosis epidemiological model, for which the authors prove the existence of non-negative and bounded global solutions. A two nodes network is analysed where the nodes represent the tuberculosis epidemiological situation of the countries Angola and Portugal. The effect of different coupling and intensity of migratory movements between the two countries and the exploration of the effect of seasonal migrations, are analysed. For a random complex network setting, the authors show that it is possible to reach a synchronization state by increasing the coupling strength and test the influence of the topology in the dynamics of the complex network. All the results are analysed through numerical simulations where the given algorithms are implemented with the python language, in a Debian/GNU-Linux environment.

\section{Estimating the Employment Band of Inaction with Multiple Breaks due to Labor Market Reforms}

\section{P.R. Mota and P.B. Vasconcelos}

This paper proposes a new way of testing the effect of labor market reforms in the employment adjustment at macro level. Following the approach to hysteresis based on the presence of non-convex costs of adjustment, a switching aggregate employment equation is estimated, with an unknown splitting factor, from a computational implementation of the linear play hysteresis operator. The play hysteresis operator describes a dynamics where nonconvex adjustment costs create intervals of weak reaction of employment to small variations in forcing variables, while spurts in employment adjustment may occur as a consequence of a large, or cumulative small shocks. The main contribution of this paper is to extend an existing approach for the case where there are disruptive breaks, by accommodating changes in the value of the switching parameter of the employment equation whenever labor market reforms are present. Results from numerical experiments on the Portuguese economy are also presented.

\section{On the $\mathbf{H}^{1}$ Conforming Virtual Element Method for Time Dependent Stokes Equation}

\section{Dibyendu Adak and Sundararajan Natarajan}

This paper presents a virtual element method for the time-dependent Stokes equation by employing a mixed formulation involving the velocity and the pressure as primitive variables. The velocity is approximated using the $\mathrm{H}^{1}$ conforming virtual element and the pressure is approximated by the discontinuous piecewise polynomial. In order to approximate the non-stationary part with optimal order of convergence, the authors need to compute the $\mathrm{L}^{2}$ projection operator of the full order $k$. In view of this requirement, they modify the velocity space keeping the same dimension. The virtual space is discrete inf-sup stable for $k \geqslant 2$ and non-divergence free. The authors also estimate the optimal order of convergence for the velocity and the pressure.

\section{Computational Simulation of the Thermal Effects on Composite Slabs Under Fire Conditions}

\section{Paulo A.G. Piloto, Carlos Balsa, Fernando Ribeiro and Ronaldo Rigobello}

A computational model is presented to evaluate the thermal effects on composite slabs with still deck, originated by standard fire exposure. Composite slabs with profiled steel deck are widely used in buildings, thus requiring fire 
resistance. Computational simulations are of great importance in this field and consist of an alternative to experimental fire tests that are expensive, time-consuming and require semi-specialized technical equipment. However, computational simulations must be reliable and realistic. The resulting transient and non-linear thermal problem is solved by the Finite Element Method in ANSYS and Matlab. The finite element models are three-dimensional, full scale, and multi-domain. Additionally, the models also include an air gap between the steel deck and the concrete part of the slab, in order to simulate the thermal effects induced by the debonding between the steel deck and the concrete, verified in previous experimental investigations. The results of the numerical simulations are validated against the results of experimental fire tests. The fire resistance of the composite slabs determined computationally is also compared with simplified calculation methods available in standards.

In a summarized way, this is the contents of the present Special Focus.

As Guest Editors, we are very pleased to have been involved in the construction of this Special Focus and in making it now available to scientific community.

We sincerely thank the authors of these contributions and also the anonymous referees who kindly volunteered their time to review these papers. Finally, we also wish to express our special thanks to the Editor-in-Chief, Professor Dongming Wang and to the Managing Editor, Professor Ilias Kotsireas for the opportunity to publish in Mathematics in Computer Science, as well as to the editorial staff, for their precious collaboration.

Publisher's Note Springer Nature remains neutral with regard to jurisdictional claims in published maps and institutional affiliations. 